\title{
Oral Candidiasis and COVID-19 in Users of Removable Dentures: Is Special Oral Care Needed?
}

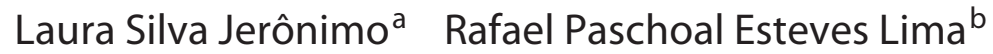 \\ Thaís Yumi Umeda Suzukic José Augusto César Discacciatic \\ Cláudia Lopes Brilhante Bhering ${ }^{c}$
}

${ }^{a}$ Faculty of Dentistry, Federal University of Minas Gerais, Belo Horizonte, Brazil; bepartment of Clinic, Pathology and Dental Surgery, Faculty of Dentistry, Federal University of Minas Gerais, Belo Horizonte, Brazil; 'Department of Restorative Dentistry, Faculty of Dentistry, Federal University of Minas Gerais, Belo Horizonte, Brazil

\section{Keywords}

Coronavirus disease 2019 - Oral candidiasis · Stomatitis ·

Denture $\cdot$ Dental care $\cdot$ Mechanical ventilation

\begin{abstract}
Elderly patients with systemic disorders and immunocompromised patients seem to have a higher risk of developing morbidity from COVID-19. Candida albicans (C. albicans) is a potentially dangerous pathogen for these patients, especially for denture wearers with prosthetic stomatitis who require mechanical ventilation. C. albicans infection, the main candidiasis infection associated with denture wear, can complicate COVID-19 and increase the associated morbidity and mortality. Therefore, early diagnosis of $C$. albicans infection in COVID-19 patients is important to establish more effective antifungal treatment methods and prophylaxis strategies. Hospitalized COVID-19 patients should undergo an oral examination to assess their oral health, and those with poor oral health should receive the appropriate care and monitoring.

(c) 2021 S. Karger AG, Basel
\end{abstract}

(c) 2021 S. Karger AG, Base

www.karger.com/ger

\section{COVID-19 Outbreak}

An outbreak of pneumonia of unknown etiology was observed in Wuhan, China, in late December of 2019 [1, $2]$. The disease pathogen was identified as a new coronavirus called severe acute respiratory syndrome coronavirus 2 (SARS-CoV-2), and the disease was named coronavirus disease 2019 (COVID-19) [2]. Considering its high transmission rate, the health status of those who are infected, and the threat to public health, COVID-19 is now considered one of the main pathogens of the respiratory system. The World Health Organization has classified COVID-19 as a pandemic since March 11, 2020 [3].

Individuals of any age can be infected with COVID-19. However, elderly patients and those with chronic medical conditions, such as arterial hypertension, diabetes mellitus, and cardiovascular diseases, have a higher risk of developing severe symptoms [4]. Approximately 10-20\% of infected individuals develop a serious case of the disease, which is characterized by interstitial pneumonia, acute breathing difficulties, and septic shock [5]. Although var-

\footnotetext{
Karger
} 
ious factors that lead to morbidity and mortality in COVID-19 have been the subject of recent research, the effects of bacterial and fungal oral coinfections have not been thoroughly investigated [6]. In addition to being more vulnerable to COVID-19, elderly people are also the most likely demographic to have fungal infections due to this group's lower immunity and higher rates of systemic diseases (e.g., diabetes mellitus) [7].

\section{Is the Oral Cavity Relevant in the Morbidity of Individuals Infected with SARS-CoV-2?}

Dental prostheses can become a reservoir for microorganisms [8], and the prevalence of elderly people who use dental prostheses is generally high [9]. In 2010, $2.3 \%$ of the global population, representing 158 million people worldwide, was edentate [9]. In Brazil, the prevalence of severe tooth loss in 2010 was significantly higher than the global mean of approximately $78.2 \%$ [10]. Oral microorganisms are associated not only with the development of oral diseases, such as denture stomatitis caused by Candida, periodontitis, or caries [8], but they can also lead to an increased risk for several systemic diseases, including aspiration pneumonia, gastrointestinal infection, pleural infection, and bacterial endocarditis [8]. In addition, infectious processes in the oral cavity can induce a systemic inflammatory process and thus affect various organs and pathologies.

The use of full or partial dental prostheses induces constant friction in the oral mucosa, increasing the proliferative ability of microorganisms. This, in turn, can lead to oral candidiasis, also known as denture stomatitis [8], which is a fungal infection that affects most users of dental prostheses (partial or total) and can be exacerbated by factors such as poor hygiene, prosthetic material, prolonged use, night use, immunosuppression, and the fungal species involved [8]. Candida-associated denture stomatitis has been found in approximately $60 \%$ of prosthesis wearers [10]. The main strain of Candida responsible for these infections is Candida albicans (C. albicans) [11].

The rate of Candida infection in elderly patients who use maxillary complete dentures can be as high as $65 \%$ $[12,13]$. This is because the prosthesis covering the mucosa produces a microenvironment conducive to Candida growth (i.e., low pH and anaerobic) [13]. Factors that promote infection include enhanced adherence of Candida spp. to acrylic, reduced saliva flow under the surfaces of the denture fittings, improperly fitted dentures, poor oral hygiene, and a high carbohydrate diet [14].

Oral Candidiasis and COVID-19 in Users

of Removable Dentures
Fungi of the genus Candida are considered opportunistic microorganisms and are known to be a potential cause of nosocomial infections [6], which can aggravate pre-existing conditions [13]. Hospitalized patients show a stronger predisposition to oral candidiasis due to environmental and systemic changes which alter the natural microbiota and favor the emergence of opportunistic infections [15]. To date, little attention has been given to the occurrence of opportunistic fungal infections in patients suffering from COVID-19 and the associated potential complications.

Patients affected by SARS-CoV-2 are subject to develop lymphocytopenia. These patients are also likely to undergo several treatments which can further increase their susceptibility to infection, such as intensive care unit (ICU) hospitalization, use of broad-spectrum antibiotics and corticosteroids, and intubation. Any preexisting chronic diseases (diabetes, hypertension, etc.) render these patients further immunocompromised [6]. Together, these factors make SARS-CoV-2 patients particularly susceptible to the development of oral and oropharyngeal candidiasis (OPC).

Although Candida pneumonia in immunosuppressed patients is rare, the presence of Candida in the respiratory tract of immunosuppressed patients should not be disregarded because it may lead to increased morbidity or mortality [16]. Colonization of the lower respiratory tract in mechanically ventilated immunocompetent patients has been shown to be associated with a longer duration of mechanical ventilation, increased pneumonia risk associated with ventilation, and increased length of stay in the ICU and the hospital, and increased mortality [16-18].

In 2018, Pendleton et al. [16] showed that Candida spp. are commonly observed in respiratory specimens of hospitalized patients, with $C$. albicans being responsible for $>78 \%$ of cases. A recent study collected samples from patients intubated in an ICU to detect possible associations between respiratory pathogens aspirated from the trachea and oral biofilm [19]. The authors reported that $59.37 \%$ of the patients exhibited the same pathogens in their tracheal aspirate and oral biofilm. Of these, $42.1 \%$ developed ventilator-associated pneumonia (VAP), and $5.2 \%$ developed aspiration pneumonia. There was a significant association between the presence of microorganisms in the tracheal and oral samples, and C. albicans was observed in $>75 \%$ of the respiratory specimens [16].

Individuals who develop severe COVID-19 may require mechanical ventilation. One study reported that $16 \%$ of COVID-19 patients are admitted to the ICU, and $8.3 \%$ require invasive ventilation [4]. Mechanical ventila- 
Table 1. Oral candidiasis characteristics

\begin{tabular}{|c|c|c|c|}
\hline Type & Clinical characteristics & Symptoms & Treatment \\
\hline $\begin{array}{l}\text { Acute pseudomembranous } \\
\text { candidiasis }\end{array}$ & $\begin{array}{l}\text { Yellowish-white plaques or nodules } \\
\text { Soft to gelatinous consistency } \\
\text { Occurs on the surface of the lip and buccal mucosa, hard and } \\
\text { soft palate, tongue, periodontal tissues, and oropharynx }\end{array}$ & $\begin{array}{l}\text { Sensitivity } \\
\text { Burning } \\
\text { Dysphagia }\end{array}$ & $\begin{array}{l}\text { Use of topical or } \\
\text { systemic antifungal* }\end{array}$ \\
\hline Acute atrophic candidiasis & $\begin{array}{l}\text { Symptomatic inflammatory erosions dispersed in the mucosa } \\
\text { Located, preferably, along the back of the tongue }\end{array}$ & $\begin{array}{l}\text { It can cause burning if } \\
\text { you eat acidic or hot food }\end{array}$ & $\begin{array}{l}\text { Use of topical or } \\
\text { systemic antifungal* }\end{array}$ \\
\hline Chronic hyperplastic candidiasis & $\begin{array}{l}\text { White stained or homogeneous lesions } \\
\text { Occurs on the oral mucosa or on the lateral border of the } \\
\text { tongue }\end{array}$ & - & $\begin{array}{l}\text { Use of topical or } \\
\text { systemic antifungal* }\end{array}$ \\
\hline Chronic atrophic candidiasis & $\begin{array}{l}\text { Bright red surface, with a velvety or stony appearance } \\
\text { Circumscribed or diffuse form } \\
\text { Ulcerated or not }\end{array}$ & $\begin{array}{l}\text { Pain } \\
\text { Irritation } \\
\text { Salivation disorders }\end{array}$ & $\begin{array}{l}\text { Use of topical or } \\
\text { systemic antifungal* }\end{array}$ \\
\hline Median rhomboid glossitis & $\begin{array}{l}\text { Symmetrical area } \\
\text { Occurs on the tongue anterior to the circumvented papilla } \\
\text { It is made up of atrophic filiform papillae }\end{array}$ & - & $\begin{array}{l}\text { Use of topical or } \\
\text { systemic antifungal* }\end{array}$ \\
\hline Angular cheilitis & $\begin{array}{l}\text { Erythematous fissure } \\
\text { Occurs in the labial commissures (unilateral or bilateral) } \\
\text { It is usually associated with an intraoral Candida infection }\end{array}$ & - & $\begin{array}{l}\text { Use of antifungal } \\
\text { creams and ointments }\end{array}$ \\
\hline
\end{tabular}

* The treatment of choice depends on the condition in which the patient and the disease are found.

tion apparatuses are associated with an increased risk of nosocomial pneumonia, specifically VAP [20]. This type of pneumonia is an important cause of morbidity and mortality in ICU patients $[6,19]$ and has been associated with the microbiota of the oral cavity and the oropharynx. For example, the presence of periodontitis has been shown to be associated with an increased risk of developing VAP [21]. In addition, patients with mechanical ventilatory support have a significant reduction in the flow of saliva. Because saliva is an important protective layer, changes in its flow can increase the risk of opportunistic infections.

A recent study showed that OPC is a probable cause of morbidity in severe COVID-19 patients [6]. Recently, a case of OPC in a non-severe COVID patient due to the use of empirical broad-spectrum antibiotics has also been reported [22]. The process of OPC infection begins with the simple colonization of Candida in the oral mucosa [6]. Patients may present with local discomfort, changes in taste, oral burning, glossodynia, dysphagia, and/or breathing difficulties [6]. In most cases, this opportunistic infection is acquired endogenously and develops when the host's local defenses are weakened [6]. C. albicans is the most relevant species in the development of this disease and is present in $>80 \%$ of cases [10]. Salehi et al. [6] reported that C. albicans accounted for $70.7 \%$ of isolates from COVID-19 patients who developed OPC. In addition, COVID-19 patients who were aged 50 or older were reported to be more likely to develop OPC. These results highlight the need for COVID-19 patients to undergo an oral cavity assessment to ascertain the presence of oral candidiasis in order to establish a treatment protocol and minimize the chances of developing Candida-related problems.

Considering the disease progression of COVID-19 and its severity, most infected patients have one or more of the several systemic (e.g., lymphocytopenia, ICU admission, invasive or noninvasive ventilation, use of broad-spectrum antibiotics, use of corticosteroids, immunosuppression) or local risk factors (e.g., use of dental prostheses, inadequate hygiene habits, reduced salivary flow due to the use of medication) that favor Candida proliferation and infection. In cases for which treatment is not performed or is ineffective, for example, in fluconazole-resistant Candida or in immunocompromised patients, oral candidiasis can spread from the oropharynx to the esophagus or systematically through the bloodstream or upper gastrointestinal tract. The resulting candidemia has been shown to have significant morbidity and a mortality rate of $71-79 \%$ [23]. It is therefore important to consider the treatment management of these patients in the hospital environment.
Jerônimo/Esteves Lima/Suzuki/ Discacciati/Bhering 


\section{How Can the Risks of Candidiasis in Hospitalized Patients Be Reduced?}

The timely detection of oral candidiasis, prosthetic stomatitis, or OPC and the identification of etiologic agents in COVID-19 patients are important to optimize therapeutic results [6]. It is important that doctors, nurses, and professionals who care for elderly patients be aware of the risk factors, diagnosis, and treatment options for oral candidiasis [13].

Oral candidiasis can present either as an acute form, as acute pseudomembranous candidiasis, and acute atrophic candidiasis (or erythematosus) or as a chronic form, as chronic hyperplastic candidiasis (or Candida leukoplakia), chronic atrophic candidiasis (candidiasis induced by prosthesis or prosthetic stomatitis), median rhomboid glossitis, and angular cheilitis. The characteristics of the different types of oral candidiasis are shown in Table 1. Pseudomembranous candidiasis and prosthesis-induced candidiasis (prosthetic stomatitis) are the most common forms. The plaques present in pseudomembranous candidiasis are easily removed, revealing an erythematous and non-ulcerated mucosa [13]. In most cases, this form of the disease has asymptomatic lesions, except in the most severe cases where patients complain of sensitivity, burning, and dysphagia [13]. In prosthetic stomatitis, the palate is hyperemic and painful. However, most patients are asymptomatic [13]. Clinical symptoms are reported in Table 1.

The drugs indicated for the treatment of C. albicans infections are topical antifungals, such as nystatin (in the form of lozenges, suspensions, or mouthwashes) and should be used as a rinse 4 times a day for 2 weeks, or disinfectant agents such as 0.12 or $0.2 \%$ chlorhexidine [24]. However, systemic antifungal therapy is recommended in patients with recurrent intolerance to topical treatment and patients at high risk of developing systemic infections. In these cases, 2 drugs can be used: clotrimazole, in the form of an oral tablet or $10 \mathrm{mg}$ pills, administered 5 times a day for 14 days, or fluconazole, at a dosage of 50$100 \mathrm{mg}$ daily for 7-14 days, or in the case of stomatitis prosthetic, a dosage of $50 \mathrm{mg}$ for 14 days, administered concomitantly with local antiseptic treatment for the prostheses [13]. Nystatin is recommended as the drug of choice due to its effectiveness, absence of serious side effects by oral use, and reduced cost compared to other drugs [25]. For uncomplicated oral candidiasis where systemic treatment is necessary, topical therapy should continue, as it reduces the dose and duration of the necessary systemic treatment [13].

Oral Candidiasis and COVID-19 in Users

of Removable Dentures
All hospitalized patients should undergo an examination of the oral cavity regardless of the reason for hospitalization. However, for immunosuppressed patients or those at risk of becoming immunosuppressed, such as COVID-19 patients, examination of the oral cavity is even more important to reduce morbidity and mortality from opportunistic infections such as prosthetic candidiasis. For people who wear prostheses, a good starting point would be to collect the patient's oral anamnesis before removing the prostheses and performing a complete mouth examination, including an inspection of the soft and hard palate as well as the oral mucosa [13]. In cases where uncomplicated oral candidiasis is identified, improved oral hygiene and topical antifungals are generally suitable treatments.

It is important that the dentist adequately supervises and guides nurses and nursing technicians to perform satisfactory and effective oral hygiene. The hygiene protocol can be easily performed by professionals from the ICU multidisciplinary team. Oral hygiene in ICU patients is a basic and necessary procedure, objective of which is to maintain tissue health of the oral cavity. This procedure aims to prevent infections, maintain mucosal moisture, promote patient comfort, and prevent the development and maturation of a biofilm with higher pathogenicity in the oral cavity, which includes not only teeth but also mucosa, tongue, and fixed and/or mobile prosthetic devices [26]. The use of broad-spectrum antibiotics to treat VAP patients has increased fungal infection in treated individuals [19]. This emphasizes the importance of establishing guidelines about Candida colonization and assessment of invasive candidiasis and ultimately establishes early antifungal treatment according to the diagnosis obtained [19].

Patients at potential risk of complication due to oral candidiasis, such as hospitalized COVID-19 patients, must be submitted to a strict oral hygiene protocol. The teeth, oral cavity, tongue, and dentures must be cleaned daily. The oral cavity's teeth and mucous surfaces should be cleaned daily with a soft brush or a gauze soaked with 0.12 or $0.2 \%$ chlorhexidine [27]. The prostheses must be cleaned and disinfected daily and removed from the oral cavity at night or for at least $6 \mathrm{~h}$ a day [10]. Isolates of C. albicans from the surface of the dentures produce a higher amount of proteinases and phospholipases, which increases their potential to cause infection [28]. Mechanical cleaning of the prosthesis is highly recommended: brushing 3 times a day with a soft brush and non-abrasive toothpaste, and immersing it weekly in $0.5 \%$ sodium hypochlorite solution (only for dentures that do not have metallic surfaces) or $0.12 \%$ chlorhexidine for $10 \mathrm{~min}$. These steps are the most effective methods to clean removable dentures [29]. When

Gerontology 2022;68:80-85

DOI: $10.1159 / 000515214$ 
using topical antifungals, dental prostheses must be removed to allow contact between the mucosa and the antifungal. The antifungal must be kept in the mouth for a few minutes to contact all mucous surfaces [13].

Considering that patients admitted to the ICU often have poor oral hygiene, protocols such as the use of $0.12 \%$ chlorhexidine can be implemented. Antiseptics reduce oropharyngeal colonization of gram-negative and gram-positive microorganisms as well as the incidence of VAP and fungal infections [30]. Chemical control of oral pathogens with $0.12 \%$ chlorhexidine appears to be more effective in preventing VAP than mechanical removal by brushing alone [27]. However, more studies on the relationships between oral candidiasis and OPC in critically ill COVID-19 patients with prostheses should be performed to establish effective antifungal prophylaxis and treatment strategies.

\section{Conclusion}

Based on the evidence presented, C. albicans infection, the main candidiasis infection associated with dentures, can negatively impact COVID-19 patients by increasing the associated morbidity and mortality. Hospital staff can benefit from a greater awareness of the negative effects that unsatisfactory oral health can have on COVID-19 patients. The findings discussed in this report highlight the importance of having oral health service providers present in ICUs to ensure proper care for elderly patients with dental prostheses as well as other immunologically vulnerable COVID-19 patients. In addition, dentists and dental hygienists need to focus more on edentulous pa- tients who use removable prostheses to improve oral hygiene habits in those patients.

\section{Acknowledgements}

Thanks to the Pró-Reitoria de Pesquisa (PRPq) of the Universidade Federal de Minas Gerais (UFMG) for financial assistance.

\section{Statement of Ethics}

This article does not contain any studies with human participants or animals performed by any of the authors.

\section{Conflict of Interest Statement}

The authors have no conflicts of interest to declare.

\section{Funding Sources}

The work received no financial support.

\section{Author Contributions}

All authors contributed to the study conception and design. Material preparation, data collection, and analysis were performed by Laura Silva Jerônimo, Cláudia Lopes Brilhante Bhering, and Rafael Paschoal Esteves Lima. The first draft of the manuscript was written by Laura Silva Jerônimo and all authors commented on previous versions of the manuscript. Thaís Yumi Umeda Suzuki and José Augusto César Discacciati critically reviewed the article. All authors read and approved the final manuscript.

\section{References}

1 Bogoch II, Watts A, Thomas-Bachli A, Huber C, Kraemer MUG, Khan K. Pneumonia of unknown aetiology in Wuhan, China: potential for international spread via commercial air travel. J Travel Med. 2020;27(2):taaa008.).

$2 \mathrm{Lu} \mathrm{H}$, Stratton CW, Tang YW. Outbreak of pneumonia of unknown etiology in Wuhan, China: the mystery and the miracle. J Med Virol. 2020;92(4):401-2.

3 Rothan HA, Byrareddy SN. The epidemiology and pathogenesis of coronavirus disease (COVID-19) outbreak. J Autoimmun. 2020;109: 102433.

4 Guan WJ, Ni ZY, Hu Y, Liang WH, Ou CQ, $\mathrm{He}$ JX, et al. Clinical characteristics of coronavirus disease 2019 in China. N Engl J Med. 2020;382(18):1708-20.

5 Huang C, Wang Y, Li X, Ren L, Zhao J, Hu Y, et al. Clinical features of patients infected with
2019 novel coronavirus in Wuhan, China. Lancet. 2020;395(10223):497-506.

6 Salehi M, Ahmadikia K, Mahmoudi S, Kalantari S, Jamalimoghadamsiahkali S, Izadi A, et al. Oropharyngeal candidiasis in hospitalised COVID-19 patients from Iran: species identification and antifungal susceptibility pattern. Mycoses. 2020;63(8): 771-8.

7 Bianchi CM, Bianchi HA, Tadano T, Paula CR, Hoffmann-Santos HD, Leite DP Jr, et al. Factors related to oral candidiasis in elderly users and non-users of removable dental prostheses. Rev Inst Med Trop Sao Paulo. 2016;58(17):17

8 Baba Y, Sato Y, Owada G, Minakuchi S. Effectiveness of a combination denturecleaning method versus a mechanical method: comparison of denture cleanli- ness, patient satisfaction, and oral healthrelated quality of life. J Prosthodont Res. 2018;62(3):353-8.

9 Kassebaum NJ, Bernabé E, Dahiya M, Bhandari B, Murray CJ, Marcenes W. Global burden of severe tooth loss: a systematic review and meta-analysis. J Dent Res. 2014 Jul;93(7 Suppl):20S-8S.

10 Azevedo JS, Azevedo MS, De Oliveira LJ, Correa MB, Demarco FF. Needs for dental prostheses and their use in elderly Brazilians according to the national oral health survey (SBBrazil 2010): prevalence rates and associated factors. Cad Saúde Pública. 2017;33(8): e00054016. [in Portuguese]

11 Salerno C, Pascale M, Contaldo M, Esposito V, Busciolano M, Milillo L, et al. Candida-associated denture stomatitis. Med Oral Patol Oral Cir Bucal. 2011;16(2):e139-43. 
12 Moraes EN, Viana LG, Resende LMH, Vasconcellos LS, Moura AS, Menezes A, et al. COVID-19 in long-term care facilities for the elderly: laboratory screening and disease dissemination prevention strategies. Ciên Saúde Colet. 2020;25(9):3445-58.

13 Akpan A, Morgan R. Oral candidiasis. Postgrad Med J. 2002;78(922):455-9.

14 Gulati M, Nobile CJ. Candida albicans biofilms: development, regulation, and molecular mechanisms. Microbes Infect. 2016;18(5): 310-21.

15 Santos SBD, Sabadin CES, Mario DN, Rigo L, Barbosa DA. Presence of Candida spp. and candidiasis in liver transplant patients. An Bras Dermatol. 2018;93(3):356-61.

16 Pendleton KM, Dickson RP, Newton DW, Hoffman TC, Yanik GA, Huffnagle GB. Respiratory tract colonization by Candida species portends worse outcomes in immunocompromised patients. Clin Pulm Med. 2018; 25(6):197-201.

17 Sachdev M, Ready D, Brealey D, Ryu J, Bercades G, Nagle J, et al. Changes in dental plaque following hospitalisation in a critical care unit: an observational study. Crit Care. 2013;17(5):R189.

18 Delisle MS, Williamson DR, Albert M, Perreault MM, Jiang X, Day AG, et al. Impact of Candida species on clinical outcomes in patients with suspected ventilator-associated pneumonia. Can Respir J. 2011;18(3):131-6.
19 Souza LCD, Mota VBRD, Carvalho AVDSZ, Corrêa RDGCF, Libério SA, Lopes FF. Association between pathogens from tracheal aspirate and oral biofilm of patients on mechanical ventilation. Braz Oral Res. 2017;31(e38): e38-9.

20 Safdar N, Crnich CJ, Maki DG. The pathogenesis of ventilator-associated pneumonia: its relevance to developing effective for prevention. Respir Care. 2005;50(6):725-41. Available from: http://rc.rcjournal.com/content/50/6/725/tab-pdf.

21 Jerônimo LS, Abreu LG, Cunha FA, Esteves Lima RP. Association between periodontitis and nosocomial pneumonia: a systematic review and meta-analysis of observational studies. Oral Health Prev Dent. 2020;18(1):11-7.

22 Riad A, Gad A, Hockova B, Klugar M. Oral candidiasis in non-severe COVID-19 patients: call for antibiotic stewardship. Oral Surg. 2020.

23 Antinori S, Milazzo L, Sollima S, Galli M, Corbellino M. Candidemia and invasive candidiasis in adults: a narrative review. Eur J Intern Med. 2016;34:21-8.

24 Ardizzoni A, Pericolini E, Paulone S, Orsi CF, Castagnoli A, Oliva I, et al. In vitro effects of commercial mouthwashes on several virulence traits of Candida albicans, viridans streptococci and Enterococcus faecalis colonizing the oral cavity. PLoS One. 2018;13(11): e0207262.
25 Williams DW, Kuriyama T, Silva S, Malic S, Lewis MA. Candida biofilms and oral candidosis: treatment and prevention. Periodontol 2000. 2011;55(1):250-65.2011

26 Alhazzani W, Smith O, Muscedere J, Medd J, Cook D. Toothbrushing for critically ill mechanically ventilated patients: a systematic review and meta-analysis of randomized trials evaluating ventilator-associated pneumonia. Crit Care Med. 2013;41(2):646-55.

27 Vilela MC, Ferreira GZ, Santos PS, Rezende NP. Oral care and nosocomial pneumonia: a systematic review. Einstein. 2015;13(2):2906.

28 Mothibe JV, Patel M. Pathogenic characteristics of Candida albicans isolated from oral cavities of denture wearers and cancer patients wearing oral prostheses. Microb Pathog. 2017;110:128-34.

29 Valentini-Mioso F, Maske TT, Cenci MS, Boscato N, Pereira-Cenci T. Chemical hygiene protocols for complete dentures: a crossover randomized clinical trial. J Prosthet Dent. 2019;121(1):83-9.

30 Liao YM, Tsai JR, Chou FH. The effectiveness of an oral health care program for preventing ventilator-associated pneumonia. Nurs Crit Care. 2015;20(2):89-97. 